\title{
Hacia unA NUEVA Historiografía. De LA Historia DEL PRESENTE A LA FILOSOFíA CON GENTE ADENTRO
}

\section{Towards a New Historiography. From the History of the Present to PhILOSOPHY WITH PEOPLE IN}

\author{
Martin J. Larsson* \\ DOI: http://dx.doi.org/10.29043/liminar.v18il.731
}

Resumen: En México existe actualmente un intento por ofrecer más apoyo institucional al estudio sobre la "historia del presente", una historiografía que se revela como una alternativa a la historia atada al pasado, y con ello como una nueva promesa para la disciplina. En este artículo argumento que esta corriente no logra percatarse de algunas herencias del tipo de historiografía que critica, por lo que tampoco logra formular una alternativa efectiva. Para encontrar una opción que rompa con esa herencia, propongo un acercamiento a las historiografías que enfatizan la importancia del encuentro y de las conexiones entre actores.

Palabras clave: historia del tiempo presente; posestructuralismo; pragmatismo; hermenéutica; estudios subalternos.

Abstract: In México, an effort is currently being made to offer improved institutional support to the "history of the present" - a historiography touted as an alternative to history constrained by the past, and thus as a new approach for the discipline. In this article, I argue that this perspective fails to perceive its own legacy from the historiography that it criticizes, since it also fails to propose an effective alternative. To find an alternative that breaks with this heritage, I suggest an approach that lies closer to the historiographies that emphasize the importance of the encounter and the connections between actors.

Keywords: history of the present time; poststructuralism; pragmatism; hermeneutics; actor-subaltern studies.

\footnotetext{
* Martin J. Larsson. Doctor en Antropología Social por la Universidad de Mánchester, Reino Unido. Profesor en el Departamento de Antropología de la Universidad de las Américas Puebla, México. Temas de especialización: antropología organizativa, antropología de los negocios, conocimientos prácticos, conocimientos de expertos, política cotidiana. Correo electrónico: martin.larsson@outlook.es. ORCID: https://orcid.org/0000-0002-8996-3772.
}

Enviado a dictamen: 9 de abril de 2019.

Aprobación: 19 de junio de 2019.

Revisiones: 1 


\section{Introducción}

¿E s posible la historia del presente en México? Es la pregunta que discutieron los conferencistas magistrales del Coloquio Internacional para una Historia del Presente en México organizado por la Universidad Nacional Autónoma de México en octubre de 2013, como parte de un esfuerzo por ubicar la historiografía de este tipo en el país. ${ }^{1}$ Desde entonces, se han organizado otros eventos y la historia del presente ha despertado cierto interés entre historiadores en el país.

Como argumentó la organizadora del evento, Eugenia Allier Montaño, lo que buscan los historiadores del presente - a grandes rasgos y enfocándonos más en temas comunes que en las diferencias- es una ruptura con aquella historiografía que ha sido confinada al estudio del pasado. Un aspecto que destaca esta autora es que la historiografía que defiende se mueve en un periodo histórico en transición, que por lo tanto no es posible "cerrar" (Allier, 2018:103). ${ }^{2}$ Al mismo tiempo, expresa una inclinación por delimitar una época contemporánea que habría iniciado en 1989 con la caída del muro de Berlín y el fin de la guerra fría, una delimitación que comparte con muchos otros historiadores del presente. ${ }^{3}$ Este presente, argumenta, constituye tanto el objeto de estudio como el escenario donde actúa el historiador (Allier, 2018:103; Modonesi, 2008:248), y es la característica principal que diferencia a los historiadores del presente de otros historiadores. Aparte de los puntos destacados por Allier Montaño, es común que en la historia del presente se resalte su énfasis en la historia oral y su inclinación por considerar la memoria misma como su principal producto, sobre todo para delimitarla frente a otras disciplinas. ${ }^{4}$ La idea es que la memoria, como lo expresa Peter Winn, forma parte de "una lucha por el pasado, librada en el presente para dar forma al futuro" (Winn, 2007:13-14).

La historia del presente se inscribe en una tradición que se formó en varios países alrededor de 1950 (Delacroix, 2018:9) y que, en un inicio, se centraba en el estudio de la Segunda Guerra Mundial. Su forma contemporánea tiene más que ver con una serie de ideas que surgieron en relación con el movimiento estudiantil de 1968, sobre todo en París, y sus lazos con diversos movimientos que tomaron forma alrededor de la década de 1960, desde los movimientos en contra del colonialismo hasta el feminismo y los grupos de reivindicación gay. La historia del presente que se practicó en aquel entonces buscaba ligarse a estos actores, escribiendo "historias alternativas" que pudieran formar parte de sus proyectos de transformación social. ${ }^{5}$

Esta herencia de una historiografía ligada al activismo político es otro aspecto importante de la historia del presente. Los conferencistas magistrales del coloquio ${ }^{6}$ son buenos ejemplos de este deseo de insertarse en discusiones políticas contemporáneas. Sus investigaciones incluyen la publicación de historias que no han tenido cabida en las representaciones oficiales de la guerra civil en Perú (Milton, 2014a); una discusión teórica sobre el proceso de "subjetivación política" en la tradición marxista (Modonesi, 2014); una descripción de la lucha por la memoria de Pinochet en Chile (Winn, 2007), y una historia política de la Ciudad de México "desde su fundación hasta el año 2000” (Rodríguez, 2012).

Si bien es cierto que los estudios de este tipo han mostrado que la historia del presente es "posible" —contestando así a la pregunta que formuló Allier Montaño para el coloquio al cual he hecho referencia-, en realidad su problema es otro. No pienso aquí en las críticas que algunos historiadores del presente consideran como las más comunes: las que se enfocan en que la historia de esta naturaleza, al no estudiar eventos "terminados", presenta mayores restricciones de accesibilidad a los archivos relevantes y carece de una falta de distancia cronológica (Delacroix, 2018:13-14). Lo que quiero destacar en este artículo es que la historia del presente se interesa más en un posicionamiento político en conflictos contemporáneos que en los propios fundamentos teóricos. Por su énfasis en la temporalidad se pierde de vista la importancia de las discusiones teóricas o filosóficas básicas para la investigación académica. Deseo sugerir en este sentido que los historiadores del presente utilizan ideas ligadas 
al cientificismo evolucionista para ubicarse tanto a sí mismos como a sus objetos de estudio en una época particular. Con eso, la propuesta de hacer una historia del presente significa un retorno a viejos problemas, más que una salida a problemas historiográficos contemporáneos. Voy a argumentar que la salida de esos problemas tiene que incluir una ruptura con los esquemas de la historiografía cientificista para abrir paso a una historiografía que se podría describir como una "filosofía con gente adentro" (Ingold, 1992 y 2017; Aróstegui, 2001:18).

El texto empieza con una breve revisión sobre diferentes maneras de conceptualizar lo contemporáneo, tomando como ejemplo la discusión reciente en torno al concepto de Antropoceno, que se puede entender como un intento por escribir una historia del presente, si bien no se ha hecho por parte de los historiadores de este tipo. Señalo los fundamentos de este pensamiento y los problemas que conlleva, para después proponer que la historia del presente no logra capturar lo más inquietante de la historiografía contemporánea. Esto me lleva a preguntar: ¿cómo formular una nueva historiografía que no repita los problemas clásicos que encontramos en términos como época y sociedad? Si bien existen varios intentos en este sentido, me voy a centrar en la propuesta de Michel Foucault por su influencia fundamental tanto en la historiografía como en otras disciplinas. Enfatizo su idea de construir una historia alternativa desde los márgenes y las fisuras del estructuralismo. Posteriormente señalo cómo esta propuesta depende del modelo que critica, por lo que su alternativa es bastante reducida. En el último apartado presento una estrategia inspirada en el pragmatismo, y otra en la hermenéutica fenomenológica, que muestran una posibilidad de formular una historiografía que no solo evade los problemas del estructuralismo, señalados por Foucault y otros, sino también los del posestructuralismo foucaultiano.

\section{El Antropoceno y la idea de época}

Los historiadores del presente desde luego no son los únicos autores que han argumentado que estamos en una nueva época. Si bien existe cierto consenso en utilizar 1989 como el inicio de la era del presente, durante los últimos años ha habido una verdadera competencia por proponer el término más adecuado para esta época. La competencia a la que me refiero empezó con el término de "Antropoceno", propuesto por el químico Paul Crutzen y el biólogo Eugene Stoermer (2000). Estos autores argumentaron que las emisiones de dióxido de carbono, el exterminio de especies, el aumento dramático de la ocupación humana de la tierra, etcétera, son procesos de tal envergadura que los seres humanos se tendrían que ver como una fuerza geológica que ha sido fundamental para la formación física del mundo contemporáneo, tan fundamental que el planeta ya habría dejado atrás el Holoceno. Tras esta propuesta, no solo se ha discutido el inicio del Antropoceno, si se tendría que ligar a la revolución neolítica (Ruddiman, 2003), si tuvo lugar durante la revolución industrial (Crutzen, 2002) o si el cambio significativo más bien ocurrió a mediados del siglo XX (Steffen et al., 2015). T También se han sugerido términos alternativos para la supuesta época contemporánea. Así, Malm y Hornborg (2014) propusieron el término Capitaloceno para resaltar el papel fundamental del capitalismo en los cambios climáticos, al mismo tiempo que este último término evitaría la homogenización del término de Antropoceno, al implicar que diferentes grupos e individuos no tienen la misma responsabilidad por la situación contemporánea. También podríamos mencionar otros términos propuestos como Econoceno (Norgaard, 2013), Tecnoceno (Malm y Hornborg, 2014:67) y Plantacionoceno (Haraway et al., 2015), todos los cuales enfatizan diferentes temas considerados centrales para el mundo contemporáneo: la economía, la tecnología y las plantaciones.

Esta discusión sobre el Antropoceno tampoco es la única que presupone la idea de una historia dividida en épocas. Por varias décadas hemos visto propuestas que han intentado caracterizar "la sociedad" o "la modernidad" contemporánea. Algunas de las propuestas más influyentes incluyen la idea de una "sociedad del espectáculo" (Debord, 1995); la "sociedad del riesgo" (Bech, 1998); la "sociedad del 
conocimiento" y la "era de la discontinuidad" (ambos de Drucker, 1992); la "modernidad líquida" (Bauman, 2003); la "[condición de] la posmodernidad" (Harvey, 1990), y, más recientemente, "la sociedad del ratio" (Bornemark, 2018). ${ }^{8}$

Lo destacable de estas propuestas no es solo que cuestionan el consenso de los historiadores del presente sobre que el año 1989 marcaría una nueva época. Más interesante es en realidad su uso de un modelo que no se ha despegado de la idea evolucionista de la historia como una secuencia de épocas marcadas por cambios de la "sociedad". Si bien el evolucionismo no tiene mayor relevancia como marco interpretativo de la historia hoy en día, la misma idea de épocas dialoga con el evolucionismo. En otras palabras: a pesar de quitar la direccionalidad teleológica que formaba parte del evolucionismo, al hacer referencia a diferentes épocas se utiliza una metáfora anclada en el evolucionismo, donde no cabe más que un concepto capaz de caracterizar cada etapa evolutiva. El problema evidente es que no es fácil señalar una sola causa, con un solo término, que pueda explicar el mundo en un momento dado y que, además —como Malm y Hornborg (2014:67) esperan- pueda llevar a acciones capaces de resolver los problemas centrales de ese tiempo. Regresando a las propuestas que he señalado arriba, ies el Capitaloceno realmente el término más adecuado para el mundo contemporáneo, dada la fascinación de los antiguos países socialistas por el progreso industrial? ¿Y por qué utilizar el capitalismo como punto de partida en lugar de la ética protestante, como lo sugiere Weber (2001[1930])? Por otro lado, si hablamos en términos del Plantacionoceno, ¿no corremos el riesgo de perder de vista el proyecto intelectual más amplio en el que podría ubicarse: el proyecto del ratio - el conteo dentro de marcos establecidos (ver nota 3 y la discusión sobre el término más abajo) - como base del conocimiento científico? Hablar de la sociedad del ratio, en turno, ino exagera la importancia de las ideas, restando valor, por ejemplo, a los modos de producción? Desde la perspectiva de los historiadores del presente, habría que agregar preguntas sobre tendencias políticas internacionales, su relación con historias locales, etcétera.
Aparte de este problema, pensar en términos de épocas y de sociedades tiene implicaciones que es importante considerar para la forma de imaginarse tanto el objeto como el objetivo de estudio; como formuló Donna Haraway, "importa qué historias contamos para contar otras historias; [y] qué descripciones describen descripciones" (2016:12). Las categorías de "épocas" y "sociedades" necesariamente van a contar una historia ligada al Estado, que se supone dirige "la sociedad", insertando así el estudio en la política del Estado, donde se trata de presentar los mejores argumentos para una visión particular de la sociedad en una época específica. Es decir, las mismas metáforas de época y sociedad fácilmente implican un papel del historiador como asesor o crítico del príncipe. Lo particularmente complicado de los términos época y sociedad son sus pretensiones universalistas, no por su dificultad de percibir lo individual, sino por los problemas que muestran para reconocer la validez simultánea de diferentes historias. A lo que llevan estos términos es a intentos por insertar el Antropoceno dentro de una historia del capitalismo, o el capitalismo dentro de una historia de cambios morales, etcétera, con lo que se busca girar la mirada de un aspecto "particular" a lo más "general", de modo que se resta importancia a lo "particular", es decir, a las historias que no funcionan como marco general.

\section{El pasado y la filosofía en la historiografía}

La idea de época entonces constituye un problema importante para la propuesta de la historia del presente, un problema que comparte con muchas otras propuestas. En realidad, el mismo entendimiento de épocas hace que corrientes como la historia del presente tengan problemas para leer otras propuestas historiográficas. Por el énfasis temporal de la historia del presente - que se encuentra incluso en el nombre que utilizan para distinguirse-, sus defensores pierden de vista que el asunto para muchos historiadores ha sido otro. Al revisar la historia de la historiografía, lo que aparece son más bien preocupaciones de carácter teórico o filosófico, teorías 
que la historia del presente incluso ha heredado a veces, sin necesariamente darse cuenta de ello.

Este no es el espacio para dibujar la historia de las diferentes corrientes historiográficas con mayor detalle, una historia por demás conocida entre historiadores. ${ }^{9}$ Sin embargo, considerando su lugar marginal para la historia del presente, vale la pena hacer algunos apuntes breves para señalar los problemas centrales que se han enfrentado en la historiografía durante las últimas décadas. De esa manera resultará también más sencillo pensar en cómo escribir historia hoy día.

Podemos encontrar un ejemplo significativo de la importancia teórica de la historiografía en los escritos de uno de los autores más influyentes para la historiografía en el siglo XX, sobre todo para el estructuralismo: Marc Bloch. En el centro de sus ideas encontramos un espacio teórico que se construye a partir de un par de tensiones básicas, tensiones que a veces desaparecen en lecturas contemporáneas. ${ }^{10}$ Lo que podemos ver en su Apología para la historia... es una estructura basada en dos dicotomías: entre épocas y continuidad, por un lado, y entre sociedad e individuo, por el otro. Esto es algo que resulta bastante evidente cuando Bloch introduce sus ideas sobre la historia. La palabra "historia", argumenta: "no veda ninguna dirección hacia la cual se pueda orientar la investigación: sea de preferencia hacia el individuo o hacia la sociedad, sea hacia la descripción de las crisis momentáneas o hacia la búsqueda de los elementos más durables (Bloch, 2001:53).

Este autor tampoco se inclinaba por un lado u otro de estas dicotomías: más bien intentaba juntarlos. Por ejemplo, argumentaba que se ocupaba "a la vez del estudio del hombre individual, esto es la filosofía, y del estudio del hombre social, esto es la historia" (Bloch, 2001:56, nota 4). A veces, los extremos de estas dicotomías resultaban más difíciles de conciliar. Así, expresó cierta molestia ante la separación demasiado tajante entre épocas, sobre todo cuando se trataba de distinguir el presente del pasado (Bloch, 2001:54). Como consecuencia - y al igual que los historiadores del presente- Bloch se oponía a la idea de la historiografía como una "ciencia del pasado" (2001:54), y al mismo tiempo re-instauró la idea de épocas al argumentar que sería un error pensar en la existencia de algo inmutablemente humano (2001:70); que "un fenómeno histórico nunca se explica plenamente fuera del estudio de su momento" (2001:64), y sobre todo al argumentar que "[1] os hombres se parecen más a su tiempo que a sus padres" (2001:64).

El énfasis teórico de la historiografía, que es evidente en los textos de Bloch, de hecho también puede observarse en las críticas a ciertas ideas historiográficas, incluyendo las críticas a los diversos intentos por escribir historias del presente. Si regresamos por un momento a la discusión sobre la manera de conceptualizar el presente, puede verse cómo los términos propuestos - Antropoceno, sociedad del riesgo, etcétera- enfatizan el tipo de ideas que se encuentran en el polo de época y sociedad, y cómo, por lo tanto, se abren a una crítica desde los extremos opuestos del modelo. En la crítica a la idea del Antropoceno no es extraño encontrar argumentos que enfatizan la falta de lo individual y circunstancial. Es el caso de la crítica a las pretensiones globales del término, a su reduccionismo, al riesgo que implica perder de vista muchos otros problemas que pueden ser importantes en lugares concretos, y a su incapacidad para dar cuenta de la participación desigual de la humanidad en la producción del cambio climático. ${ }^{\text {lI }}$

El hecho de que se ocupan del presente, por el otro lado, no es algo que aparece en las críticas. Desde luego, no es de sorprenderse considerando que otras disciplinas no comparten la misma obsesión con el tiempo que aparece en la historiografía. Lo que nos ayuda a ver esta ausencia, sin embargo, es que lo interesante no es la época que uno decide estudiar - como implica la crítica de la historia del presentesino el hecho mismo de pensar en épocas, y cómo implica cierta direccionalidad de las discusiones.

Esto nos lleva a la pregunta que motiva este artículo: iqué pasa si se formula una historiografía que cuestiona el uso de conceptos como época y sociedad? 


\section{La propuesta foucaultiana}

Si bien varios autores han intentado formular una historiografía que cuestione las ideas de época y sociedad, para los propósitos del presente artículo me voy a centrar en el autor que ha dejado la huella más grande en este sentido: Michel Foucault. En La arqueología del saber (Foucault, 1969), la idea de épocas fue fundamental para la crítica que formuló al estructuralismo. Lo que le estorbaba a este autor era la manera de construir generalizaciones a partir de eventos específicos que se insertan en cadenas de eventos e ideas dominantes. Esta historiografía, argumentó, ha creado monumentos que forman parte de una narrativa totalitaria en la que los actos que se "desvían" de esta narrativa son anulados, vueltos invisibles. Lo que buscaba hacer a través de su "arqueología del saber" era deconstruir los sistemas aparentemente coherentes, buscando las fisuras de los discursos y las palabras que han sido censuradas por no apoyar a las grandes narrativas. Así, sugería, se abriría un espacio para tratar los discursos más allá de las organizaciones discursivas establecidas, donde aparecería la singularidad de cada acto o cada declaración.

Este tipo de historiografía se volvió muy popular en los años ochenta, en gran parte por la publicación de Orientalism, de Edward Said (1979), una obra que fue fundamental para presentar las ideas foucaultianas al público académico en Estados Unidos (Racevskis, 2005:83). Siguiendo la metodología foucaultiana, Said mostró cómo la idea del Oriente fue creada por un círculo de europeos que se referenciaban entre ellos, lo que condujo a la producción de ciertos consensos sobre el carácter del Oriente - una imagen que se contraponía a la del europeo racional, de clase alta-.

Si la propuesta foucaultiana mostraba su fortaleza para seguir este tipo de constructos discursivos, también se utilizó para escribir historias al margen de las grandes narrativas. A veces, esto se hizo de manera bastante literal, como cuando Michael Camille (1992) escribió sobre los gestos subversivos que veía en los márgenes de los manuscritos ilustrados y sobre las figuras que se encuentran en los bordes de las iglesias medievales.
Pero también se utilizó la idea del margen para seguir a personajes que no formaban parte del canon establecido por la historiografía. Es lo que, por ejemplo, hizo Edda Manga (2003) cuando se topó con los archivos de una mujer de La Habana del siglo XVIII, cuyos textos habían terminado en un archivo de la Inquisición como parte de la prueba de su crimen contra la verdadera fe. Manga escogió tratarla con el mismo respeto y la misma seriedad con los que se suele tratar a filósofos reconocidos, como Kant y Hegel, cuestionando así el canon establecido a la vez que señalaba la posibilidad de una historia alternativa.

Vale también la pena subrayar que el tipo de historiografía que seguía la línea marcada por Foucault era muy diferente a los intentos de escribir una historia desde los subalternos, que también se puso de moda más o menos al mismo tiempo - una línea que a veces se cruza con la historia del presente, como puede observarse en los ejemplos de los conferencistas magistrales referidos en la nota 1 - ${ }^{12}$ Mientras la historiografía foucaultiana se interesaba por los márgenes del discurso dominante, los estudios subalternos se interesaban por un grupo particular dentro de uno de los modelos que ha dialogado con el evolucionismo, el modelo marxista. Si a primera vista podría parecer que las personas "marginales" serían las mismas tanto desde una perspectiva foucaultiana como desde una perspectiva marxista, la gran diferencia es que la marginalidad marxista existía dentro de un modelo preestablecido, que marcaba una tarea para este grupo — de liberar tanto al opresor como al oprimido-, mientras la marginalidad foucaultiana trataba de captar la vida fuera de los modelos.

Si esta diferencia no siempre parece muy evidente, tampoco ha facilitado la cosa que una autora como Gayatari Spivak (1988) haya escogido utilizar el término de "subalterno" para la persona marginalizada en los modelos discursivos dominantes, es decir, para la persona que no tiene voz a través de esos esquemas. ${ }^{13}$

Si los estudios inspirados en el posestructuralismo foucaultiano efectivamente han movido la historiografía hacia nuevos actores, y hacia nuevos problemas, también han mostrado que la búsqueda de lo marginal 
o "subalterno" es una empresa bastante paradójica. Por un lado, es fácil que se vuelva un tipo de mapeo, donde los márgenes constantemente se mueven. Por otro lado, el mismo planteamiento de Foucault — de buscar en las fisuras de la historiografía estructuralista - implica que los estudios de este tipo siempre van a depender del modelo que critican, dado que ese mismo modelo genera los márgenes que se buscan. Esto es bastante evidente en el modelo propuesto por Spivak, para quien el subalterno deja de ser tal cuando empieza a hablar, al mismo tiempo que el mismo encuentro con el subalterno se da en relación con el modelo donde aparece como tal. Con ello, la empresa académica se vuelve una suerte de búsqueda de diferentes voces en la que la meta inalcanzable es la igualdad y la representatividad discursiva -inalcanzable, entre otros factores, porque quien busca y recopila voces no está en la misma posición de quien es buscado y recopiladoEl problema central de este tipo de búsqueda, sin embargo, no es que implique una meta inalcanzable. Más bien consiste en la falta de una narrativa propia, lo que no depende directamente del modelo que se critica. En la búsqueda por los márgenes, las voces de los "subalternos" o marginalizados no son interesantes en primer lugar por lo que dicen, sino por la posición desde donde hablan. Por ello, aun en estos momentos sigue hablando un tipo de teoría mapeadora que solo promete una vida dentro de los marcos preestablecidos.

Si queremos dejar hablar a las personas por fuera de los modelos teóricos, tendría que hacerse a partir de un interés en lo que hacen o en lo que tienen que decir, y no por quienes son dentro de un modelo teórico. Esto, desde luego, resulta muy difícil siguiendo una propuesta como la de la historia del presente, dado que en primer lugar esta se interesa por el momento de un evento y por su importancia política, es decir, por cuestiones definidas antes del encuentro con los sujetos del estudio.

\section{Estrategias pragmáticas y hermenéuticas}

Considerando los problemas de la propuesta foucaultiana - problemas que no han sido tratados por la historia del presente-, la pregunta es cómo pensar en alternativas a la historiografía anclada en el evolucionismo sin repetir los problemas implícitos en el posestructuralismo foucaultiano. A continuación voy a esbozar algunos rasgos centrales de dos de las propuestas sobre cómo crear una narrativa propia por fuera de las ideas discutidas arriba: una que llamo "estrategia pragmática" y, la otra, "estrategia hermenéutica".

La estrategia pragmática que tengo en mente se liga en primer lugar a la extensa obra de Bruno Latour. Como han subrayado Harman (2009:13) y Hämäläinen y Lehtonen (2016:25-26), el problema fundamental de Latour se trata de una aversión a cualquier forma de reduccionismo, de allí su profundo pragmatismo. Un ejemplo de esta postura aparece en la descripción que hace de una excursión que realizó a Mont Aiguille. Latour enfatizó cómo el mapa que utilizó para subir la montaña encerraba una historia de cientos de años que le conectaba a la burocracia estatal, a relojes atómicos, a satélites, a estandarizaciones, a la invención del GPS, etcétera (Latour, 2013:89). A través de este ejemplo argumentó que el mapa no se podía entender como una representación directa de la montaña, sino como parte de una cadena que mediaba entre la montaña y el montañista. Por ello, argumentó, "la referencia no está ligada ni al sujeto cognoscente ni al objeto conocido" (Latour, 2013:79). Con eso evita el reduccionismo que implican términos como época y sociedad, al rehusar explicaciones que no consideran todos los objetos de una cadena: al cuestionar la relación dicotómica entre mapa y territorio, argumenta sobre la importancia de incluir todas las cosas que forman parte de lo que estudiamos, y no dejar que sean meras expresiones de algo más profundo. El mapa, en otras palabras, no se entiende como una representación de "la realidad", sino como parte de una cadena amplia de cosas, donde las características de cada cosa son importantes. Cada objeto, sugiere, tiene que considerarse como un "actante" en el mundo: ninguno es un sencillo "intermediador", que no agrega nada a las conexiones que se establecen. Esto es lo que enfatiza cuando sigue las conexiones entre los GPS y los satélites, etcétera. 
Lo interesante para Latour no es la correspondencia entre mapa y territorio, sino las alianzas que un nudo específico en estas redes - como Latour subiendo la montaña- puede hacer con otros objetos.

Como puede deducirse de lo anterior, la acción - en este caso la acción de subir la montaña- es algo que nunca está muy lejos para Latour, un asunto que también ha sido central para el pragmatismo en general (James, 1975[1907]; Dewey, 1995[1908]). Esta inclinación por lo práctico también se encuentra en su manera de imaginarse el uso de sus ideas. Su aspiración es hacer un texto que pueda funcionar como una "guía de viaje", que "busca ayudar [a los profesionales] una vezque están empantanados en el territorio" (Latour, 2005:34-35). El propósito para él es señalar "dónde viajar" y "qué es lo que vale la pena ver allí" (Latour, 2005:34-35).

Lo interesante de estas ideas, en el contexto que he esbozado, es que le permiten a Latour decir algo sobre el mundo, y no solo sobre los discursos sobre el mundo formulado por otros, que fue tan importante para Foucault. Latour tampoco formula una metafísica, con una ontología específica, como puede percibirse en la confianza estructuralista; más bien se acerca a la idea de una "verdad instrumental", como formularía el pragmático James (1975[1907]:44-45), es decir, un conocimiento que "funciona" (work; James, (1975[1907]:34).

La estrategia hermenéutica en turno consiste en "conversar" con personas que aparecen en los archivos: puede resultar más sencillo establecer este tipo de conversaciones si se buscan personas cuyas ideas se encuentran lejos de los modelos utilizados en la actualidad, como si fueran de un lugar exótico. ${ }^{14}$ Este tipo de historiografía hermenéutica es, por ejemplo, la que ha utilizado Jonna Bornemark (2018) cuando ha involucrado a pensadores renacentistas en la discusión sobre la fijación contemporánea en las estadísticas y la contabilidad administrativa. Conversando con Nicolás de Cusa, Bornemark recuperó un esquema que ayuda a entender esta fijación, al mismo tiempo que ofrece un concepto para pensar en una alternativa a esa forma de pensar. Lo que había hecho Nicolás de Cusa fue separar entre lo que llamaba ratio e intellectus. Mientras el ratio se mueve dentro de un objeto establecido - midiendo y calculando, por ejemplo, el calor y el frío dentro del objeto de temperatura-, el intellectus se mueve al margen del conocimiento formulando nuevos objetos. Dicho de otra manera: el intellectus se interesa por los términos que se utilizan para medir el mundo -formulando preguntas como: ¿qué quiere decir "el desarrollo"? y ¿qué cosa es "la pobreza"? - , mientras el ratio es el tipo de racionalidad que mide algo a partir de conceptos existentes - icómo mejorar los índices de pobreza?, ¿cómo mejorar la productividad agrícola de una zona particular?- - Un aspecto importante sobre este punto es que la idea de intellectus no se imagina al mundo como algo que se puede mapear de una vez por todas, mientras la racionalidad del tipo ratio presupone lo opuesto. Lo que no se conoce, desde una perspectiva de intellectus, no es algo que se puede conquistar: lo desconocido más bien es un espacio que permite nuevas formas, siempre imperfectas, de conceptualizar el mundo.

El trabajo de Bornemark (2015) sobre Matilde de Magdeburgo es otro ejemplo en este sentido. En su obra utilizó el mismo método para discutir cuestiones metafísicas, de nuevo como una manera de pensar más allá de los límites del ratio, pero también para cuestionar ciertas ideas contemporáneas sobre lo religioso. En diálogo con los textos de Matilde de Magdeburgo, Bornemark subraya aspectos de la vida que no se pueden contabilizar dentro de marcos teóricos establecidos, como el hecho de ver, escuchar, hablar, etcétera; son estos los hechos a los que Matilde de Magdeburgo llamaba "lo divino", y que para Bornemark abren un espacio por fuera de los objetos que es posible contabilizar. La pregunta que le interesa a esta autora gira entonces en torno al "hecho" de contar, y no tanto en torno a diferentes "formas" de contar. Cuando queremos tomar decisiones sobre el futuro, argumenta, el conteo y los hechos solo nos pueden ayudar hasta cierto punto. Si, por ejemplo, vamos a escoger una carrera universitaria, no basta con saber todos los hechos sobre el contenido de cada carrera; ese conocimiento puede mejorar nuestro entendimiento de las carreras 
y nos puede ayudar a determinar la plausibilidad de diferentes efectos, pero no va a ser suficiente para saber si la actividad específica es algo que vamos a disfrutar o que nos dará una sensación de sentido. Por ello, cualquier decisión tendrá que contener un aspecto de intuición. El arte que subraya entonces no consiste en la capacidad de calcular efectos, sino de actuar con cierta sabiduría, lo que va más allá de los conocimientos del tipo ratio.

A través de estos estudios, Bornemark involucra tanto a Nicolás de Cusa como a Matilde de Magdeburgo en conversaciones contemporáneas de una manera "intelectual" - es decir, de una manera ligada al conocimiento del tipo de intellectus - , y no los utiliza como ejemplos que pueden demostrar su teoría —es decir, no los utiliza como parte de un conocimiento del tipo del ratio - . Esto implica, de entrada, la confianza en que los encuentros que establece sean fructíferos para los temas que le interesan, pero también permite la posibilidad de que puedan surgir nuevos temas imprevistos de esos encuentros. Con eso, Bornemark entonces sigue un procedimiento que rompe tanto con el modelo según el cual la tensión entre individuo y la sociedad domina, como con la crítica foucaultiana que busca experiencias en las fisuras de ese modelo: de este modo, la historia se escribe a partir de un encuentro en el que el interés reside en lo que puede decir "el otro", y no en lo que sus ideas pueden expresar sobre las posiciones en un marco teórico existente.

De esta manera ha surgidoun tipo de investigaciones que se podrían entender como "una filosofía con la gente adentro", como lo formuló Tim Ingold (1992:696). Se trata de un tipo de investigación que ha dejado las pretensiones que marcaron el inicio de las ciencias sociales, es decir, las pretensiones por encontrar leyes sociales, al estilo de las leyes naturales, que podrían indicar la dirección de una evolución social y que, a su vez, podrían servir para la legislación de los Estados y para la administración pública. En su lugar se voltean nuevamente hacia preguntas "filosóficas" que se interesan por el significado de ciertos conceptos al estilo de una racionalidad del intellectus. Lo que ha subrayado Ingold es, en primer lugar, la importancia de enfatizar la naturaleza especulativa de las investigaciones, junto con su carácter abierto (open-ended), comparativo y crítico (Ingold, 2017:21-22); no se trata en primer lugar de las preguntas que tendrían que hacerse. A esto deseo agregar un fundamento inspirado en los textos aristotélicos visible sobre todo en la obra de Bornemark. En vez de pensar en una separación entre teoría y práctica —o entre mapa y territorio- se enfatiza la importancia de la experiencia para los conocimientos prácticos (frónesis); donde la "teoría" no se entiende como el resultado de la investigación, sino como la investigación misma, y donde no se separa el conocimiento abstracto del conocimiento práctico, sino que se entiende como parte del mismo episteme (Ramírez, 1997). Desde esta perspectiva, el regreso de la filosofía que sugiere Ingold, y que retomo aquí, se podría entender como un intento de repensar las ciencias sociales y las humanidades al entablar un diálogo con saberes que fueron marginalizados durante la dominación cientificista.

\section{Conclusiones y reflexiones finales}

En este artículo he revisado los postulados básicos de la historia del presente, una historiografía que actualmente ha despertado cierto interés en México. He enfatizado cómo este tipo de historiografía se presenta como un giro importante de una historiografía delimitada al estudio del pasado, hacia una que se interesa más en problemas contemporáneos. Lo que he querido enfatizar es que el aspecto temporal en sí no ha sido un asunto central para formular historiografías. Como muestra el caso de Marc Bloch, la preocupación principal que guiaba sus ideas era cómo moverse en las dicotomías época/continuidad y sociedad/individuo, preocupaciones que ubicaba en su obra, firmemente centrada en los problemas en boga desde el evolucionismo decimonónico, hasta el estructuralismo y el funcionalismo que estaban surgiendo a principios del siglo XX. En otras palabras, los problemas que le preocupaban a Bloch no giraban en torno de la temporalidad, sino en el uso de ciertas 
dicotomías. El aspecto fundamental que está ausente en los otros historiadores del presente es, entonces, cómo entienden estas dicotomías.

He argumentado que el modelo que dio lugar a las dicotomías que utilizaba Bloch ha sido central para las discusiones historiográficas desde finales de los años sesenta, impulsadas sobre todo por el trabajo de Foucault y su idea de hacer una arqueología del saber en vez de insertarse en esas dicotomías. Al mismo tiempo, he señalado que el problema de la propuesta foucaultiana es que depende del tipo de teorías que critica, al construir sus propuestas en las fisuras que se encuentran en ellas. Por ello, la historia del presente ofrece poca ayuda para abandonar esas complicaciones.

He señalado dos posibles salidas a los problemas del estructuralismo y el posestructuralismo que han sido trabajadas durante los últimos años: la salida pragmática y la salida de la hermenéutica fenomenológica. Lo interesante de estas propuestas es la manera en que crean una narrativa propia por fuera de los esquemas referidos, siguiendo las conexiones entre una red de actores, como lo hace Latour, o construyendo nuevos conocimientos a partir de encuentros, como lo hace Bornemark. He argumentado que estos autores han logrado producir este giro gracias al alejamiento radical de los fundamentos cientificistas que guiaban el trabajo de Bloch y otros, y al regreso de una filosofía más interesada en el conocimiento corporal que en propuestas inspiradas en la idea de leyes naturales. Lo que nos ofrecen autores como Latour, Bornemark e Ingold, entonces, es una salida a problemas fundamentales con los que se han estado enfrentando las ciencias sociales y las humanidades durante el siglo pasado, una salida que implica un movimiento hacia algo que se asemeja a una filosofía con la gente adentro.

Quiero sugerir que para la historiografía en México las propuestas de este tipo —que he agrupado bajo el concepto de "filosofía con la gente adentro"- pueden ayudar a pensar la historia de una nueva manera, lo que difícilmente harían las corrientes que se ligan a las ideas estructuralistas y posestructuralistas. Nos podrían, por ejemplo, ayudar a entender mejor cómo se piensan y cómo se han pensado conceptos como política, religión, desarrollo, juego, parentesco, etcétera. Más importante, nos podrían ayudar a conceptualizar procesos que todavía no tienen nombre. Con ello, lo que nos permite la filosofía con gente adentro es la formulación de nuevas preguntas cuyas respuestas no se pueden intuir de antemano; de formular, en breve, una nueva historiografía.

\section{Notas}

${ }^{1}$ Los conferencistas magistrales fueron: Alberto del Castillo, Massimo Modonesi, Cynthia Milton, Ariel Rodríguez Kuri y Peter Winn. Quien organizó el evento fue Eugenia Allier Montaño.

2 Ver también Rousso (2016).

${ }^{3}$ Ver sobre el tema: Allier (2018:103), Hartog (2015:104), Garton (2014:158), Fazio Vengoa (2010:149-168) y Aróstegui (2004:208-219).

${ }^{4}$ Ver sobre el particular: Fazio (2010:140), Rousso (2016), Milton (2014a y 2014b) y Modonesi (2008:249).

${ }^{5}$ Ver por ejemplo: Allier (2018:101-102), Aguirre Rojas (2004:78) y Delacroix et al. (2003:95-96).

${ }^{6}$ Consultar en la nota 1 la lista de conferencistas.

7 Para la discusión sobre el inicio del Antropoceno, ver también Angus (2015).

${ }^{8}$ La idea de una sociedad del ratio enfatiza la dominación del conteo para diversos modelos de gobernanza, lo que se contrapone a la exploración de nuevas maneras de pensar el mundo. Regresaré a estas ideas en la parte sobre las estrategias hermenéuticas.

${ }^{9}$ Véase por ejemplo Lloyd (2009); si bien tiende a pensar en las escuelas de una manera que las asemeja a épocas. Ver la introducción para referencias sobre historiadores que enfatizan el carácter temporal de la historia.

10 Allier Montaño, por ejemplo, argumenta que: "Los Annales surgieron en contra del positivismo científico del siglo XIX, y para oponerse a él, rechazaron lo político y el acontecimiento, centrándose en un análisis estructural" (Allier, 2018:102); como veremos, el estructuralismo que inició Bloch se interesaba también por lo individual y el acontecimiento.

${ }^{11}$ Para una revisión de este tipo de críticas, ver Haraway et al. (2015). 
12 Las investigaciones que he mencionado de los conferencistas magistrales referidos en la nota l tienen un interés marcado en historias de sectores que se podrían considerar como subalternos en el sentido gramsciano es decir, como una clase dominada-, al mismo tiempo que enfatizan la exclusión discursiva que han sufrido, que se acerca más a las preocupaciones de Foucault.

${ }^{13}$ Tampoco facilita el entendimiento de estas diferencias que Spivak (1988) haya ubicado a Foucault entre los teóricos que supuestamente establecen esquemas que no dejan espacio para otras palabras que para aquellas que hacen eco del modelo teórico utilizado por parte del analista.

${ }^{14}$ En realidad, esta propuesta tiene poco que ver con el énfasis en conexiones y rutas que encontramos en la obra de Latour, pero tiene una cercanía histórica con el pragmatismo, sobre todo a partir del trabajo de Heidegger (1977[1972]; ver también Begby, 2015). Al igual que los pragmáticos, Heidegger se distanciaba de la idea de que el conocimiento podía empezar desde una posición desligada de la experiencia cotidiana del mundo; pensaba que se construía a partir de nuestra inmersión en el mundo y nuestra relación práctica con los objetos que nos rodean. Su famoso ejemplo de la relación con un martillo es importante en este sentido. Heidegger argumentaba que nos relacionamos con el martillo ante todo usándolo, no observándolo como si fuera un objeto externo a nosotros y nuestro mundo. Esta postura no desconoce la posibilidad de un conocimiento desligado de la práctica, si bien señala que existe un riesgo con ese tipo de operación, dado que nos pueden alejar del mundo y las cuestiones que importan en nuestras vidas. Esta situación de "estaren-el-mundo" (Dasein) entonces es fundamental para nuestra manera de entender el mundo. Por ello, la estrategia hermenéutica inspirada en Heidegger se centra en el encuentro con el mundo y el sentido práctico que surge de él.

\section{Referencias}

Aguirre Rojas, Carlos Antonio (2004). La historiografía en el siglo XX: Historia e Historiadores entre 1848 y i2025? Barcelona: Montesinos.
Allier Montaño, Eugenia (2018). "Balance de la historia del tiempo presente. Creación y consolidación de un campo historiográfico". En Revista de Estudios Sociales, (65), 100112. Disponible en: https://doi.org/10.7440/res65.2018.09 Angus, Ian (2015). "When Did the Anthropocene Begin... and Why Does It Matter?". En Monthly Review, 67(4).

Aróstegui, Julio (2001). La investigación histórica: teoría y método. Barcelona: Crítica.

Aróstegui, Julio (2004). La historia vivida: sobre la historia del presente. Madrid: Alianza.

Bauman, Zygmunt (2003). Modernidad líquida. Ciudad de México: Fondo de Cultura Económica.

Bech, Ulrich (1998). La sociedad del riesgo: hacia una nueva modernidad. Barcelona, Buenos Aires y Ciudad de México: Paidós.

Begby, Endre (2015). "Hermeneutics and Pragmatism". En Jeff Malpas y Hans-Helmuth Gander (eds.), The Routledge Companion to Philosophical Hermeneutics. S/l: Routledge, pp. 612-622.

Bloch, Marc (2001). Apología para la historia o el oficio de historiador. Ciudad de México: Fondo de Cultura Económica.

Bornemark, Jonna (2015). Kroppslighetens mystik: en filosofisk läsning av Metchild von Magdeburg. Estocolmo: Volante.

Bornemark, Jonna (2018). Det omätbaras renässans: En uppgörelse med pedanternas världsherravälde. Estocolmo: Volante.

Camille, Michel (1992). Image on the Edge: The Margins of Medieval Art. Londres: Reaktion Books.

Crutzen, Paul Josef y Eugene F. Stoermer (2000). "The 'Anthropocene”. En IGBP Newsletter. 41, 17-18.

Crutzen, Paul Josef (2002). "Geology of Mankind". En Nature, 415(6867), 23.

Debord, Guy (1995). La sociedad del espectáculo. Santiago de Chile: Naufragio.

Delacroix, Christian (2018). "L'histoire du temps présent, une histoire (vraiment) comme les autres?". En Tempo \& Argumento, 10(23), 5-38. Disponible en: http:// dx.doi.org/10.5965/2175180310232018005.

Delacroix, Christian et al. (2003). Histoire et historiens en France depuis 1945. París: ADPF.

Dewey, James (1995[1908]). "Does Reality Possess Pratical Character?”. En Russel B. Goodman (ed.), 
Pragmatism: A Contemporary Reader. Nueva York y Londres: Routledge, pp. 79-91.

Drucker, Peter Ferdinand (1992). The Age of Discontinuity: Guidelines to Our Changing Society. New Brunswick y Londres: Transaction Publishers.

Fazio Vengoa, Hugo (2010). La historia del tiempo presente. Historiografía, problemas y métodos. Bogotá: Universidad de los Andes.

Foucault, Michel (1969). L'archéologie du savoir. París: Gallimard.

Garton Ash, Timothy (2014). The Magic Lantern: The Revolution of ' 89 Witnessed in Warsaw, Budapest, Berline Prage. Londres: Atlantic Books.

Hämäläinen, Nora y Turo-Kimmo Lehtonen (2016). "Latour's empirical metaphysics". En Distinktion: Journal of Social Theory, 17(1), 20-37.

Haraway, Donna et al. (2015). "Anthropologists Are Talking - About the Anthropocene". En Ethnos: Journal of Anthropology, 1-30. Disponible en: doi:http:// dx.doi.org/10.1080/00141844.2015.1105838

Haraway, Donna (2016). Staying with the Trouble: Making Kin in the Chthulucene. Londres: Duke University Press.

Harman, Graham (2009). Prince of Networks: Bruno Latour and Metaphysics. Melbourne: re.press.

Hartog, François (2015). Regimes of Historicity: Presentism and Experiences of Time. Nueva York: Columbia University Press.

Harvey, David (1990). The Condition of Postmodernity: An Enquiry Into the Origins of Cultural Change. Cambridge y Oxford: Blackwell.

Heidegger, Martin (1997[1972]). Ser y tiempo. Santiago de Chile: Editorial Universitaria.

Ingold, Tim (1992). "Editorial”. En Man, 27(4), 693-696.

Ingold, Tim (2017). "Anthropology contra Ethnography". En HAU: Journal of Ethnographic Theory, 7(1), 21-26. Disponible en: DOI: http:// dx.doi.org/10.14318/hau7.1.005

James, William (1975[1907]). Pragmatism. Cambridge: Harvard University Press.

Latour, Bruno (2005). Re-ensamblar lo social: Una introducción a la teoría del actor-red. Buenos Aires: Manantial.
Latour, Bruno (2013). Investigación sobre los modos de existencia: una antropología de los modernos. Buenos Aires, Barcelona y Ciudad de México: Paidós.

Lloyd, Christopher (2009). "Historiographic Schools". En Aviezer Tucker (ed.), A Companion to the Philosophy of History and Historiography. Oxford: Wiley-Blackwell, pp. 371-380.

Malm, Andreas y Alf Hornborg (2014). "The Geology of Mankind? A Critique of the Anthropocene Narrative". En The Anthropocene Review, 1(1), 62-69.

Manga, Edda (2003). Gudomliga uppenbarelser, demoniska samlag: en studie av det excentriska idéarvet i Cecilia Rodríguez tänkande. Gotemburgo: Glänta.

Milton, Cynthia E. (2014a). "Introduction". En Cynthia E. Milton (ed.), Art from a Fractured Past: Memory and Truth-Telling in Post-Shining Path Peru. Durham y Londres: Duke University Press, pp. 1-36.

Milton, Cynthia E. (2014b). "Images of Truth: Rescuing Memories of Peru's Internal War Through Testimonial Art". En Cynthia E. Milton (ed.), Art from a Fractured Past: Memory and Truth-Telling in Post-Shining Path Peru. Durham y Londres: Duke University Press, pp. 37-74.

Modonesi, Massimo (2008). "Historia, memoria y política: entrevista con Enzo Traverso". En Andamios, 4(8), 245-256.

Modonesi, Massimo (2014). Subalternity, Antagonism, Autonomy. Londres: Pluto Press.

Norgaard, Richard B. (2013). "The Econocene and the California Delta". En San Francisco Estuary o Watershed Science: A Broad Perspective, 11(3). Disponible en: doi:https://escholarship.org/uc/item/4h98t2m0.

Racevskis, Karlis (2005). "Edward Said and Michel Foucault: Affinities and Dissonances". En Research in African Literatures, 36(3), 83-97.

Ramírez, José Luis (1997). "La teoría del diseño y el diseño de la teoría". En Astrágalo (6), 39-52. Disponible en: https:/issuu.com/caeau/docs/ astragalo-06-final

Rodríguez Kuri, Ariel (ed.) (2012). Historia política de la ciudad de México (desde su fundación hasta el año 2000. México: Centro de Estudios Históricos-El Colegio de México. 
Ruddiman, William F. (2003). "The Anthropogenic Greenhouse Era Began Thousands of Years Ago”. En Climatic Change, 61, 261-293.

Rousso, Henry (2016). The Latest Catastrophe: History, the Present, the Contemporary. Chicago y Londres: The University of Chicago Press.

Said, Edward W. (1979). Orientalism. Nueva York: Vintage Books.

Spivak, Gayatri Chakravorty (1988). "Can the Subaltern Speak?" En Cary Nelson and Lawrence Grossberg (eds.), Marxism and the Interpretation of Culture. Campaign, Illinois: University of Illinois Press, pp. 271-313.
Steffen, Will et al. (2015). "The Trajectory of the Anthropocene: The Great Acceleration". En Anthropocene Review, 2(1), 81-98.

Weber, Max [2001[1930]). The Protestant Ethic and the Spirit of Capitalism. Abingdon y Nueva York: Routledge.

Winn, Peter (2007). "El pasado está presente. Historia y memoria en el Chile contemporáneo". En Anne Pérotin-Dumon (dir.), Historizar el pasado vivo en América Latina. S/d, pp. 1-48. Disponible en: http:// etica.uahurtado.cl/historizarelpasadovivo/es contenido.php 